\title{
Recent Development of Technology and Application of Photoacoustic Molecular Imaging Toward Clinical Translation
}

\author{
Jaesok Yu*1,2, Ho Nhu Y. Nguyen*3, Wiendelt Steenbergen ${ }^{3}$, and Kang Kim ${ }^{1,2,4}$ \\ ${ }^{1}$ Center for Ultrasound Molecular Imaging and Therapeutics, University of Pittsburgh Medical Center, Pittsburgh, Pennsylvania; \\ ${ }^{2}$ Department of Bioengineering, University of Pittsburgh, Pittsburgh, Pennsylvania; ${ }^{3}$ Biomedical Photonic Imaging Group, Faculty of \\ Science and Technology, University of Twente, Enschede, The Netherlands; and ${ }^{4}$ McGowan Institute for Regenerative Medicine, \\ University of Pittsburgh Medical Center, Pittsburgh, Pennsylvania
}

The deep imaging capability and optical absorption contrast offered by photoacoustic imaging promote the use of this technology in clinical applications. By exploiting the optical absorption properties of endogenous chromophores such as hemoglobin and lipid, molecular information at a depth of a few centimeters can be unveiled. This information shows promise to reveal lesions indicating early stage of various human diseases, such as cancer and atherosclerosis. In addition, the use of exogenous contrast agents can further extend the capability of photoacoustic imaging in clinical diagnosis and treatment. In this review, the current state of the art and applications of photoacoustic molecular probes will be critically reviewed, as well as some spearheading translational efforts that have taken place over the past 5 years. Some of the most critical barriers to clinical translation of this novel technology will be discussed, and some thoughts will be given on future endeavors and pathways.

Key Words: photoacoustic; molecular imaging; endogenous; exogenous; contrast agents

J Nucl Med 2018; 59:1202-1207

DOI: 10.2967/jnumed.117.201459

\section{$\mathbf{P}$}

hotoacoustic imaging (PAI) has been shown to be a promising medical imaging technology. This technique is based on the photoacoustic effect by which ultrasound waves are generated because of light absorption in the tissue's chromophores. Therefore, endogenous chromophores and exogenous contrast agents can be used to provide excellent contrast in photoacoustic molecular imaging. In a previous review, applications of photoacoustic molecular imaging of cancer in vivo using endogenous chromophores and exogenous contrast agents were discussed (1). Over the past few years, the technology has been extensively applied to numerous human diseases. Diagnosis of various diseases

\footnotetext{
Received Feb. 28, 2018; revision accepted May 25, 2018.

For correspondence or reprints contact: Kang Kim, University of Pittsburgh, 3550 Terrace St., Scaife Hall, Room 623, Pittsburgh, PA 15261.

E-mail: kangkim@pitt.edu

Wiendelt Steenbergen, University of Twente, Zuidhorst ZH263, P.O. Box

217, Enschede, 7500 AE, The Netherlands.

${ }^{*}$ Contributed equally to this work.

Published online May 31, 2018.

COPYRIGHT (c) 2018 by the Society of Nuclear Medicine and Molecular Imaging.
}

at an early stage through screening, as well as monitoring of treatment, is possible using PAI with endogenous chromophores. In addition, novel approaches to the use of photoacoustic contrast agents have been significantly investigated to enhance contrast, targeting, and therapeutics over traditional probes for molecular theranostics. This review focuses on recent research on photoacoustic molecular imaging exploiting endogenous chromophores and exogenous contrast agents.

\section{PAI WITH ENDOGENOUS CHROMOPHORES}

Endogenous chromophores with significantly different absorption spectra produce high endogenous contrast in PAI. Specific wavelengths can be selected to obtain maximum contrast. Furthermore, PAI can take advantage of near-infrared light at wavelengths in the range $650-1,200 \mathrm{~nm}$ for deep imaging. Angiogenesis, the formation of new blood vessels, is one of the basic hallmarks of various diseases such as cancer (2). Therefore, cancer can be revealed with PAI of hemoglobin, one of the major chromophores. As an example, Figure 1 shows maximum-intensity-projection photoacoustic images of a 40-y-old woman's breasts after elimination of superficial blood vessels from the images. In this section, we focus on PAI uses that are close to becoming clinically applicable.

\section{Breast Cancer}

Breast cancer is the leading cause of cancer death in women globally. In 2012, 0.5 million women worldwide died of breast cancer, accounting for $15 \%$ of all cancer deaths in women (3). Because angiogenesis is the marker of cancer, imaging with light at wavelengths that are mainly absorbed by the chromophores deoxy- and oxyhemoglobin can be exploited to distinguish between malignant and normal breast tissue and to monitor therapy (2).

Heijblom et al. used the Twente photoacoustic mammoscope to investigate breast cancer lesions in 31 patients (4), with 32 of 33 malignancies being visualized with high imaging contrast. On average, the lesions were seen with higher contrast on photoacoustic images than on x-ray mammography. Moreover, photoacoustic contrast was independent of mammographic breast density, whereas with $\mathrm{x}$-ray mammography there was a significant decrease in 


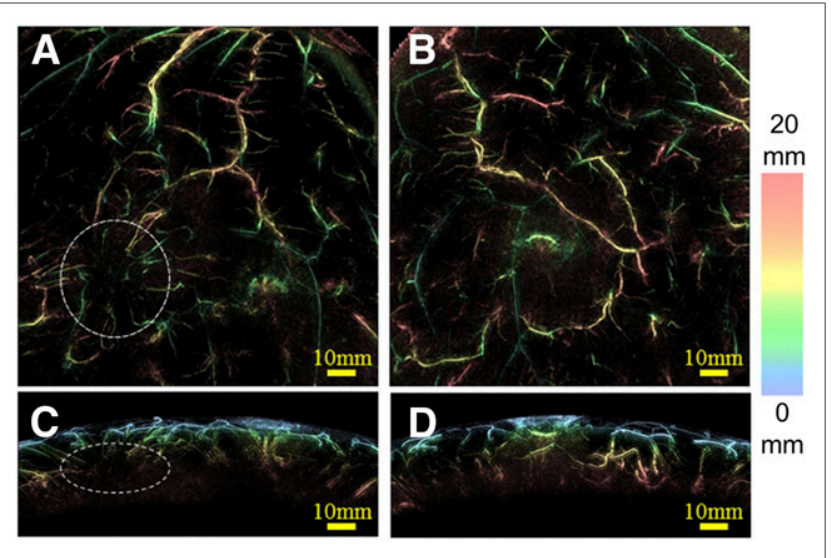

FIGURE 1. PAI with depth color-coding of 40-y-old woman's breasts. Top view (A) and side view (C) show tumor and surrounding blood vessels (encircled), indicating angiogenesis. (B and D) Top view (B) and side view (D) of contralateral breast show no lesion. (Reproduced with permission of (5).)

contrast in the low-density group compared with the highdensity group.

A new photoacoustic mammography system offering remarkably more morphologic and structural details of blood vessels than can be seen with MRI was reported by Toi et al. (5). Additionally, combining these techniques allows visualization of both the tumor mass and its related vasculature in one image, shown in their study on 22 malignancies. By using 2 wavelengths (755 and $795 \mathrm{~nm}$ ) for imaging, the investigators could estimate the oxygen saturation of hemoglobin $\left(\mathrm{sO}_{2}\right)$. This information would give insight on the tumor microenvironment and facilitate monitoring of treatment such as chemotherapy and anti-HER 2 treatment.

\section{Crohn Disease}

Crohn disease is an inflammatory bowel disease often leading to severe injuries or even death. The inflammation can be revealed by measuring hemoglobin and oxygenated hemoglobin content in the intestinal wall. Knieling et al. proposed a noninvasive approach to distinguish active and nonactive Crohn disease by determining the hemoglobin level in the intestinal wall with multispectral photoacoustic tomography (6). They performed multispectral photoacoustic tomography on 108 Crohn disease patients with 6 wavelengths and extracted total oxygenated and deoxygenated hemoglobin and $\mathrm{sO}_{2}$ in the intestinal wall. They found significant differences between active and nonactive Crohn disease for all multispectral photoacoustic tomography values excluding $\mathrm{sO}_{2}$ when using endoscopy and histologic characteristics as references.

\section{Rheumatoid Arthritis}

Rheumatoid arthritis is a chronic inflammatory disease that causes progressive destruction of affected joints leading to severe disability and even death. Conventional radiography is the technique currently used for detection of joint damage. However, radiography cannot detect the disease in the early stage (7). PAI, therefore, can be exploited because hypervascularization and angiogenesis are the hallmarks of the early stage.

van den Berg et al. observed large differences in photoacoustic images of inflamed, healthy, and noninflamed proximal interphalangeal finger joints $(8)$. Figure 2 represents their setup and some results. They used a handheld probe with cost-efficient integrated diode lasers (Fig. 2A) and a compact PAI system for imaging finger joints (Fig. 2B). A larger number of high-photoacoustic pixels (indicating the presence of blood) was observed in the photoacoustic images of inflamed joints (Fig. 2C) than in the images of noninflamed joints (Fig. 2D). Figure 2E shows their quantification study on the finger joints of 7 healthy volunteers and 10 rheumatoid arthritis patients. It appeared that inflamed

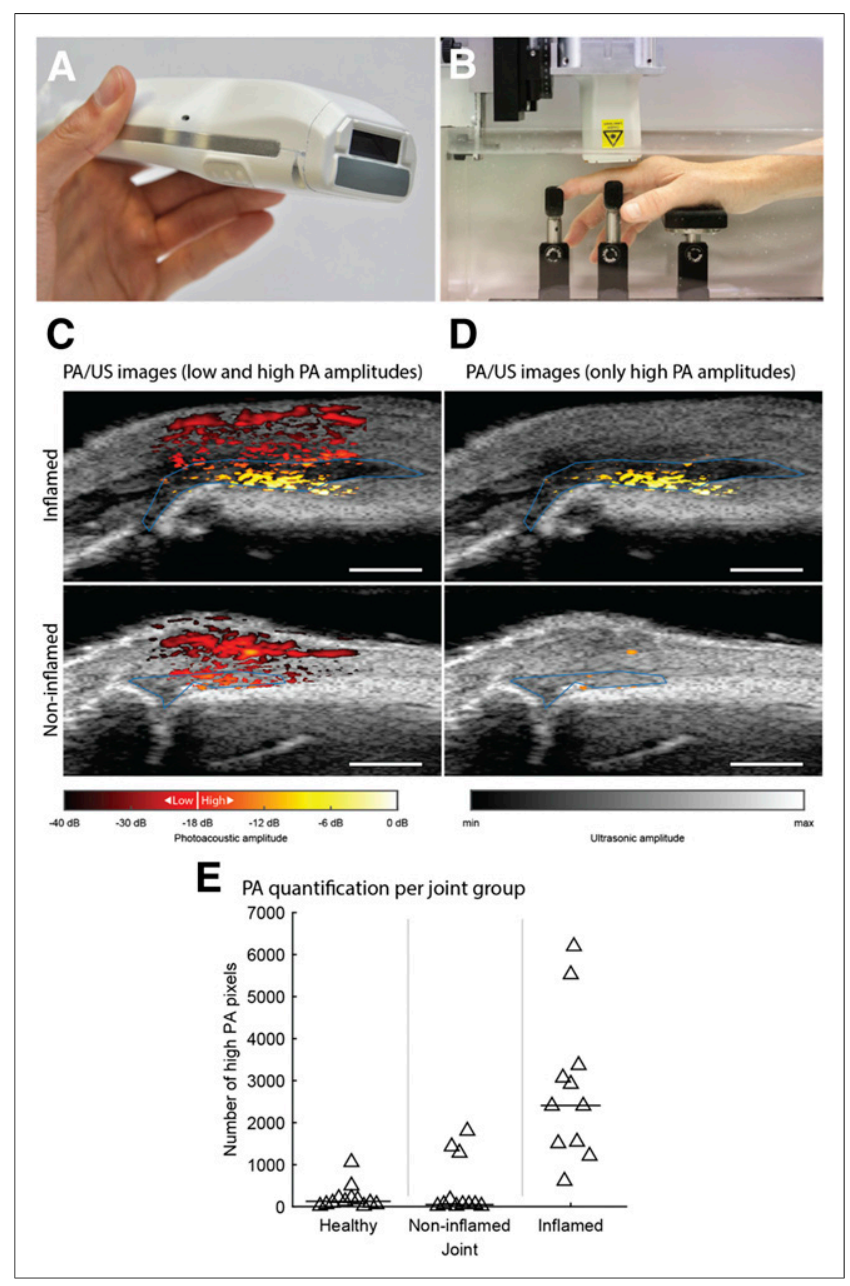

FIGURE 2. (A) Handheld probe with integrated diode lasers. (B) Finger joint PAl setup. (C) Combined photoacoustic and ultrasound images of inflamed and noninflamed finger joints. (D) Processed images with only high photoacoustic amplitudes. (E) Photoacoustic quantification study on healthy volunteers and rheumatoid arthritis patients. PA = photoacoustic; US = ultrasound. (Reproduced with permission of (8).) 
joints have significantly higher image values than noninflamed joints.

Jo et al. enhanced van den Berg et al.'s observation with their clinical research on rheumatoid arthritis patients $(8,9)$. With a photoacoustic-ultrasound dual-imaging system, they could evaluate the increased hemoglobin content, exposing hyperemia, in arthritic joints over normal joints. In addition, they performed PAI using 2 wavelengths (576 and $584 \mathrm{~nm}$ ) to quantify $\mathrm{sO}_{2}$ in each joint. Their results showed a recognizable decrease in $\mathrm{sO}_{2}$, representing hypoxia, in arthritic joints compared with normal joints. Another report from this group showed the potential of using PAI to monitor the treatment process of arthritic joints (10).

\section{Psoriasis}

Psoriasis is an incurable inflammatory skin disease. Many noninvasive imaging techniques have been proposed to facilitate the study of psoriatic skins. However, they are limited by the imaging depth, contrast, or resolution $(11,12)$. Aguirre et al. presented an ultra-broadband raster-scan photoacoustic mesoscopy system that could visualize the skin up to subdermis layer with a lateral resolution of about $20 \mu \mathrm{m}$ (12). Ultra-broadband raster-scan photoacoustic mesoscopy implemented in a hand-held probe allowed them to study vascular and melanin structures in healthy and psoriatic patients, reflecting the clinical potential of the technique. Images from 6 psoriasis patients showed that the blood volume per skin surface, the fractal number of the vasculature, and the epidermal thickness were all higher in psoriatic skin than in healthy skin. In addition, developing ultra-fast lasers will enable multispectral measurements for ultra-broadband raster-scan photoacoustic mesoscopy that could enhance the skin examination by showing oxygenation and differentiation of the melanin contributions.

\section{Prostate Cancer}

Prostate cancer is the second most common male cancer, close behind lung cancer. In 2012, 1.1 million men had prostate cancer, representing $15 \%$ of all cancers in men during that year (3). Removing the tumor without affecting the nearby neurovascular bundles required for sexual potency is the goal of radical prostatectomy. Success strongly depends on the surgeon's experience. In addition, screening for angiogenesis can be used to detect the tumor. For those purposes, hemoglobin was exploited as an endogenous chromophore by Horiguchi et al. (13) and Ishihara et al. with a PAI system combining photoacoustic and ultrasound imaging (14). They were able to visualize the periprostatic microvasculature and a photoacoustic signal pattern indicating cancer. Their handheld probe with real-time imaging shows promise for assisting surgeons during radical prostatectomy.

\section{Atherosclerotic Plaque}

Vulnerable atherosclerotic plaque can lead to cardiovascular events, which are the leading cause of death globally (15). Inside arteries, substances such as fat and cholesterol from the blood can accumulate in plaques. Lipids, therefore, are used as an endogenous contrast agent to reveal the plaque's vulnerability. Imaging lipid, however, is complicated by the blood's strong absorption. Wavelengths of maximum absorption by lipids are around 1,200 and 1,700 nm.

Not all lipids indicate plaques. Jansen et al. could differentiate between plaque lipids and periadventitial lipids, which can be found in the wall of a normal artery $(16,17)$. They showed that these types of lipids have slightly different absorption spectra. Using a combined intravascular photoacoustic and ultrasound imaging system, with multiple wavelengths near 1,200 and $1,700 \mathrm{~nm}$, they were able to distinguish periadventitial lipids and lipids in the plaque in human coronary arteries ex vivo. Catheter-based intravascular photoacoustic-intravascular ultrasound imaging systems can display images in real time, enhancing this technique's advantages for clinical applications (18).

\section{PHOTOACOUSTIC MOLECULAR IMAGING WITH EXOGENOUS CONTRAST AGENTS}

Exogenous photoacoustic contrast agents have been extensively studied in the past few years to provide molecular information on diseases with enhanced contrast (1). Clinically approved fluorescent agents with low quantum yields such as sodium fluorescein, IRDye $800 \mathrm{CW}$, methylene blue, and indocyanine green have been repurposed for contrast-enhanced PAI in preclinical studies, but only one clinical study has been reported so far in which methylene blue was used to photoacoustically detect sentinel lymph node in breast cancer (19). Besides these dyes, there have been continuous collective efforts to improve overall photoacoustic contrast, imaging depth, and specificity to the biomarkers, which would extend the capability of PAI for better diagnosis and treatments and might foster clinical translation. In this section, recent approaches to developing photoacoustic theranostic agents will be discussed.

\section{Exogenous Contrast Agents for Contrast-Enhanced, Deep-Tissue, and Multimodal PAl}

Although ultrasonically triggered phase-transition droplets as ultrasound contrast agent were discussed in the previous review, in this review optically triggered phase transition droplets as a promising photoacoustic contrast agent with superior efficiency are discussed (1,20,21). Most photoacoustic contrast agents generate signals through thermal expansion, which is known to have relatively low photoacoustic conversion efficiency (21). The perfluorocarbon family is often used to form the droplet core for its low bulk boiling temperature, such as perfluoropentane (boiling point, $29^{\circ} \mathrm{C}$ ). Once the liquid core is encapsulated by a thin layer of lipid, polymer, or protein, the vaporization temperature increases because of the increased Laplace pressure so that it maintains the liquid at physiologic temperature (22). These shelled droplets contain chromophores that increase temperature locally on light absorption (Figs. 3A and 


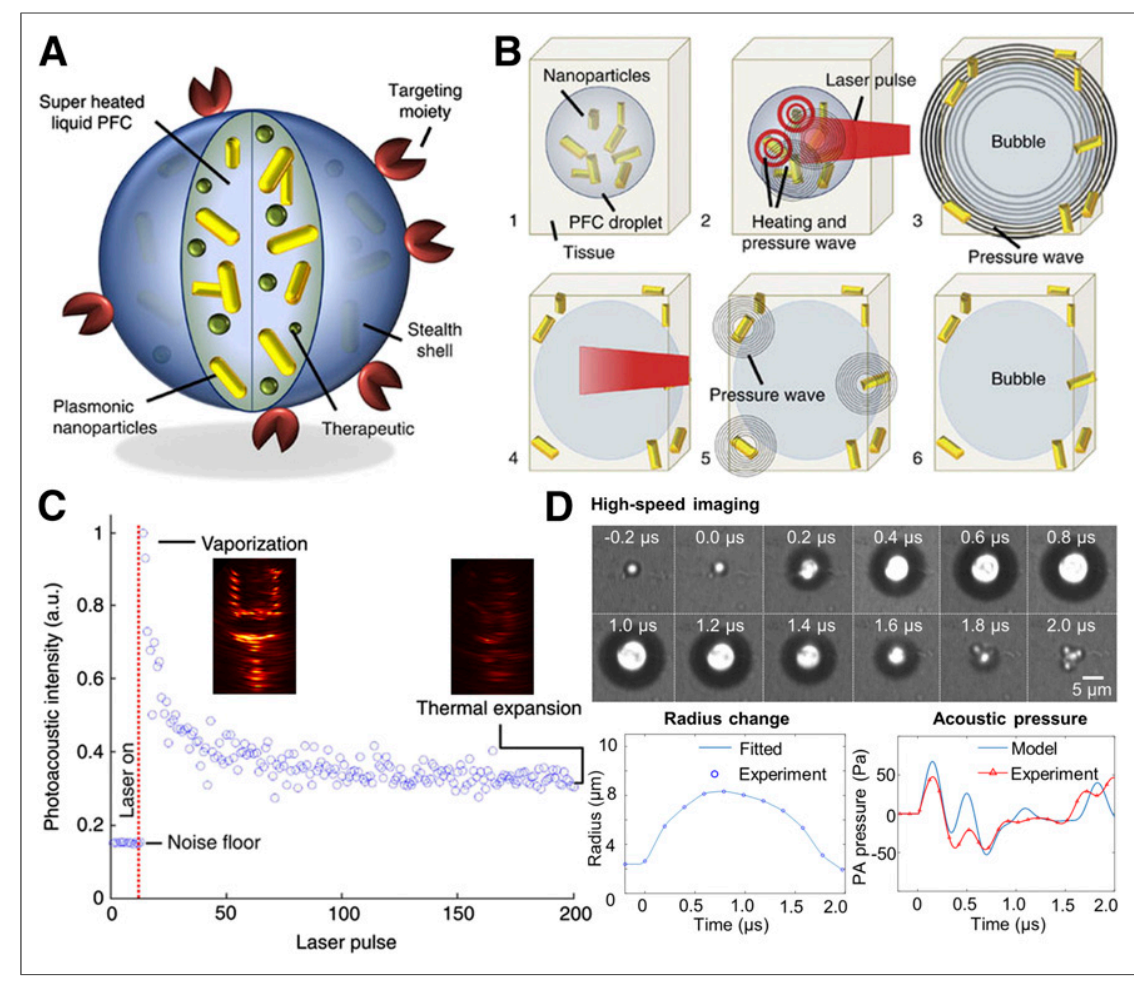

FIGURE 3. (A) Conceptual structure of optically triggered phase transition droplet. (B) Illustration of optical droplet vaporization. (C) Photoacoustic signal enhancement using vaporization compared with thermal expansion. (D) Comparison between theoretic model and concurrent high-speed imaging and acoustic measurements of optical droplet vaporization. a.u. = arbitrary units; PFC = perfluorocarbon. (Reproduced with permission of $(20,22)$.)

3B). The resulting rapid volume expansion of the shelledconstruct generates strong signals, providing significantly enhanced contrast compared with thermal expansion-based photoacoustic signals (Figs. 3B and 3C) $(20,22)$. With therapeutics inside and targeting molecules on the surface, these droplets can serve as disease-specific theranostic molecular probes (Fig. 3A). Furthermore, activated droplets that turn into gas bubbles can be used for contrast-enhanced ultrasound imaging. In this way, these droplets serve as dual-mode contrast agents for combined photoacoustic and ultrasound imaging $(20,23)$. Recently, some efforts were initiated to unveil underlying physics of optically triggered droplets using theoretic models and experimental observations. These observations and findings so far may lead to the design of droplets with optimal parameters for in vivo use, which include a balance between photoacoustic conversion efficiency and safety, and may provide a strong motivation to further develop droplets and evaluate them for use as a theranostic nanoplatform conjugated with targeting and therapeutic molecules (Fig. 3D) $(20,22,24)$.

Relatively deep imaging is a key advantage of PAI over conventional optical imaging technologies. To maximize PAI depth, Zhou et al. suggested the use of phosphorus phthalocyanine with a long wavelength absorption beyond
$1,000 \mathrm{~nm}$ to reduce light scattering, enhancing imaging depth. They successfully demonstrated PAI at depths of $11 \mathrm{~cm}$ in chicken breast tissue and $5 \mathrm{~cm}$ through human arms (25). Also, surfactant-stripped naphthalocyanines developed by Zhang et al. were used for multimodal (ultrasound, photoacoustic, and PET) functional imaging in the gastrointestinal tract of a mouse (26).

\section{Molecular Targeting Approach for PAI}

Passive targeting relevant to oncology is based on size-dependent accumulation in solid tumors because of enhanced permeability and retention caused by leaky vasculature and poor lymphatic drainage in tumors. For active targeting, identifying the biomarkers that should be overexpressed on the target is required. As a targeting ligand, several structures are used, including small molecules, peptide/ adhirons, Affibody, aptamer, and antibody/protein (27). Some efforts were made to use these targeting ligands to achieve a molecular PAI.

Zhang et al. engineered a cystineknot peptide, $\mathrm{R}_{0} 1$, labeled with Atto 740 dye and evaluated it using $\alpha_{v} \beta_{6^{-}}$ positive (A431) and $\alpha_{v} \beta_{6}$-negative (293T) tumors in mice (28). Significant photoacoustic signal enhancement was found in A431 tumors over $4 \mathrm{~h}$ after injection (Figs. 4A-4C). Levi et al. showed that their developed peptide, AA3G-740, successfully binds to gastrin-releasing peptide receptor in mouse prostate cancer, improving photoacoustic signal almost 2-fold compared with the control agent (29). Sano et al. suggested an antiepidermal growth factor receptor monoclonal antibody labeled with indocyanine green to target cancer associated with epidermal growth factor receptor. The dye highly accumulated on epidermal growth factor receptor-positive (A431 and MDAMB-468) compared with -negative tumors (T47D) (30). These technologies to simultaneously image both vascular networks by endogenous contrast and molecular features by exogenous contrast will enable molecular PAI for diagnosis and photoacoustic guided biopsy.

\section{Laser-Activated Therapy with Guidance of PAI}

In recent years, PAI was used for guiding laser-activated therapy, photothermal therapy, and photodynamic therapy in preclinical research. Functional and molecular features of tissue shown by PAI, such as vasculature, hemodynamics, functional connectivity, melanoma, and temperature, have been used for monitoring treatment efficacy (31). In addition, PAI has potential for monitoring drug distribution and concentration (31). 


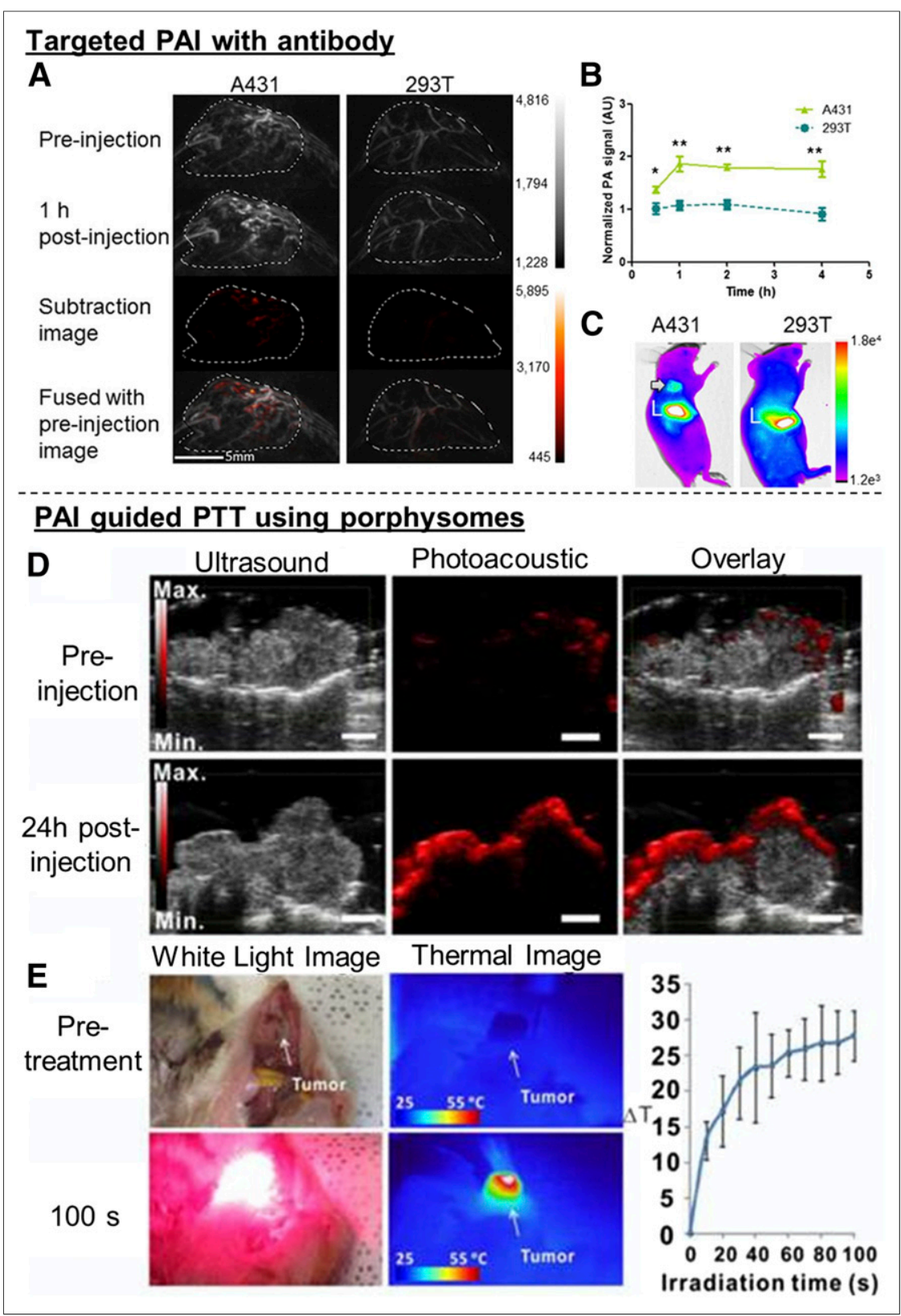

FIGURE 4. (A and B) PAl of A431 tumor with antibody targeting of $a_{v} \beta_{6}$ integrin. (C) Fluorescence imaging at $1 \mathrm{~h}$ after injection of $A 740-R_{0} 1$. Arrow points to $A 431$ tumor. $\mathrm{L}=$ liver. (D) Photoacoustic detection of hamster oral tumor at $24 \mathrm{~h}$ after injection of porphysomes. (E) Bright-field and thermal image of tumor before and $100 \mathrm{~s}$ after porphysome-enabled photothermal therapy. a.u. = arbitrary units; PA = photoacoustic; $\mathrm{PTT}=$ photothermal therapy. (Reproduced with permission of $(28,33)$.)

Photothermal therapy is localized thermal ablation using photothermal-conversion agents. Because the laser source can be shared, the synergetic ability for concurrent imaging and therapy has potential to monitor the accumulation of agents on-site during photothermal therapy. Zhang et al. successfully demonstrated treatment of a mouse tumor using terrylenediimide-based agents under PAI guidance (32). The agents showed photothermal conversion efficiency of up to $40 \%$, with peak absorption at 640-690 $\mathrm{nm}$, which is well suited for both photothermal therapy and PAI. Muhanna et al. showed photothermal therapy with porphysomes, organic nanoparticles developed for fluorescence and photoacoustic dualmodality imaging (33). Porphysomeenabled photothermal therapy allowed for PAI-monitored target-specific ablation of buccal and tongue carcinomas in hamster, and its progress was monitored by PAI (Figs. 4D and 4E).

Photosensitizers for photodynamic therapy require oxygen to generate toxic singlet oxygen that kills the target cells. Near-infrared light is desired for laser activation at sufficient depths while also allowing for deep PAI. Yu et al. demonstrated PAI with a clinical photosensitizer, verteporfin, on a mouse tumor (34). Ho et al. evaluated the photoacoustic efficacy of 5 different photosensitizers (35). Zinc phthalocyanine exhibited the most efficient photoacoustic response, and in vivo feasibility was shown using a mouse tumor model.

\section{CONCLUSION AND FUTURE DIRECTION}

PAI with advanced multifunctional theranostic agents has great potential as a noninvasive and nonionization biomedical molecular imaging and treatment tool. In addition to advanced molecular probes, clinical acceptance will increase with the advent of faster, more compact, affordable, and clinically friendly systems. One big barrier is the laser. A Q-switched solid-state laser for a typical PAI system is bulky and costly, and the wavelength-tuning speed on the optical-parametric-oscillator is too slow to achieve real-time multispectral PAI. Recently, Schwarz et al. (36) introduced a PAI system with a high repetition rate $(100 \mathrm{~Hz})$ and a fast wavelength-tuning speed $(10 \mathrm{~ms})$. In addition, there have been efforts to develop affordable PAI systems, such as replacing the bulky solidstate lasers with diode lasers or light-emitting diodes and integrating the light source and the detector into a handheld probe $(37,38)$. In efforts to make PAI more suitable for freehand scanning in a clinical environment, a concavemirror-shaped device attached to the ultrasound probe was proposed to not only protect an operator and a patient from unwanted exposure to the reflected laser light but also 
improve the signal-to-noise-ratio for deep tissue imaging (34). Exogenous photoacoustic contrast agents also should be further evaluated under biologically relevant conditions to assess bioeffects and improve binding or treatment efficiency in situ. All these approaches will bring PAI closer to clinical application. Continued efforts to develop clinically friendly systems and novel multifunctional photoacoustic contrast agents and to validate them in human subjects will accelerate the clinical introduction of photoacoustic technologies.

\section{DISCLOSURE}

This work was supported by the European Commission's H2020 program under grant 731771 . No other potential conflict of interest relevant to this article was reported.

\section{REFERENCES}

1. Wilson KE, Wang TY, Willmann JK. Acoustic and photoacoustic molecular imaging of cancer. J Nucl Med. 2013;54:1851-1854.

2. Hanahan D, Weinberg RA. Hallmarks of cancer: the next generation. Cell. 2011;144:646-674.

3. Stewart BW, Wild CP, eds. World Cancer Report 2014. Geneva, Switzerland: World Health Organization; 2014.

4. Heijblom M, Piras D, van den Engh FM, et al. The state of the art in breast imaging using the Twente Photoacoustic Mammoscope: results from 31 measurements on malignancies. Eur Radiol. 2016;26:3874-3887.

5. Toi M, Asao Y, Matsumoto Y, et al. Visualization of tumor-related blood vessels in human breast by photoacoustic imaging system with a hemispherical detector array. Sci Rep. 2017;7:41970.

6. Knieling F, Neufert C, Hartmann A, et al. Multispectral optoacoustic tomography for assessment of Crohn's disease activity. N Engl J Med. 2017;376:1292-1294.

7. Backhaus M, Kamradt T, Sandrock D, et al. Arthritis of the finger joints: a comprehensive approach comparing conventional radiography, scintigraphy, ultrasound, and contrast-enhanced magnetic resonance imaging. Arthritis Rheum. 1999;42: $1232-1245$.

8. van den Berg PJ, Daoudi K, Bernelot Moens HJ, Steenbergen W. Feasibility of photoacoustic/ultrasound imaging of synovitis in finger joints using a point-ofcare system. Photoacoustics. 2017;8:8-14.

9. Jo J, Xu G, Cao M, et al. A functional study of human inflammatory arthritis using photoacoustic imaging. Sci Rep. 2017;7:15026.

10. Rajian JR, Shao X, Chamberland DL, Wang X. Characterization and treatment monitoring of inflammatory arthritis by photoacoustic imaging: a study on adjuvantinduced arthritis rat model. Biomed Opt Express. 2013;4:900-908.

11. Lacarrubba F, Pellacani G, Gurgone S, Verzì AE, Micali G. Advances in noninvasive techniques as aids to the diagnosis and monitoring of therapeutic response in plaque psoriasis: a review. Int J Dermatol. 2015;54:626-634.

12. Aguirre J, Schwarz M, Garzorz N, et al. Precision assessment of label-free psoriasis biomarkers with ultra-broadband optoacoustic mesoscopy. Nat Biomed Eng. 2017; 1:0068.

13. Horiguchi A, Tsujita K, Irisawa K, et al. A pilot study of photoacoustic imaging system for improved real-time visualization of neurovascular bundle during radical prostatectomy. Prostate. 2016;76:307-315.

14. Ishihara M, Shinchi M, Horiguchi A, et al. Possibility of transrectal photoacoustic imaging-guided biopsy for detection of prostate cancer [abstract]. In: Oraevsky AA, Wang L V., eds. Proceedings of the SPIE. Vol 10064. Bellingham WA: International Society for Optics and Photonics; 2017: 100642U.

15. WHO Fact Sheet No. 317: Cardiovascular Diseases (CVDs). Geneva, Switzerland: World Health Organization; 2017.
16. Jansen $\mathrm{K}$, Wu M, van der Steen AFW, van Soest G. Lipid detection in atherosclerotic human coronaries by spectroscopic intravascular photoacoustic imaging. Opt Express. 2013;21:21472.

17. Jansen K, Wu M, van der Steen AFW, van Soest G. Photoacoustic imaging of human coronary atherosclerosis in two spectral bands. Photoacoustics. 2013;2: $12-20$.

18. Wu M, Springeling G, Lovrak M, et al. Real-time volumetric lipid imaging in vivo by intravascular photoacoustics at 20 frames per second. Biomed Opt Express. 2017;8:943-953.

19. Garcia-Uribe A, Erpelding TN, Krumholz A, et al. Dual-modality photoacoustic and ultrasound imaging system for noninvasive sentinel lymph node detection in patients with breast cancer. Sci Rep. 2015;5:15748.

20. Wilson K, Homan K, Emelianov S. Biomedical photoacoustics beyond thermal expansion using triggered nanodroplet vaporization for contrast-enhanced imaging. Nat Commun. 2012;3:618.

21. Tam AC. Applications of photoacoustic sensing techniques. Rev Mod Phys. 1986; 58:381-431.

22. Yu J, Chen X, Villanueva FS, Kim K. Vaporization and recondensation dynamics of indocyanine green-loaded perfluoropentane droplets irradiated by a short pulse laser. Appl Phys Lett. 2016;109:243701.

23. Yoon H, Yarmoska SK, Hannah AS, Yoon C, Hallam KA, Emelianov SY. Contrastenhanced ultrasound imaging in vivo with laser-activated nanodroplets. Med Phys. 2017;44:3444-3449.

24. Lajoinie G, Gelderblom E, Chlon C, et al. Ultrafast vapourization dynamics of laser-activated polymeric microcapsules. Nat Commun. 2014;5:3671.

25. Zhou Y, Wang D, Zhang Y, et al. A phosphorus phthalocyanine formulation with intense absorbance at $1000 \mathrm{~nm}$ for deep optical imaging. Theranostics. 2016;6: 688-697.

26. Zhang Y, Jeon M, Rich LJ, et al. Non-invasive multimodal functional imaging of the intestine with frozen micellar naphthalocyanines. Nat Nanotechnol. 2014;9: $631-638$.

27. Weber J, Beard PC, Bohndiek SE. Contrast agents for molecular photoacoustic imaging. Nat Methods. 2016;13:639-650.

28. Zhang C, Kimura R, Abou-Elkacem L, Levi J, Xu L, Gambhir SS. A cystine knot peptide targeting integrin $\alpha v \beta 6$ for photoacoustic and fluorescence imaging of tumors in living subjects. J Nucl Med. 2016;57:1629-1634.

29. Levi J, Sathirachinda A, Gambhir SS. A high-affinity, high-stability photoacoustic agent for imaging gastrin-releasing peptide receptor in prostate cancer. Clin Cancer Res. 2014;20:3721-3729.

30. Sano K, Ohashi M, Kanazaki K, et al. In vivo photoacoustic imaging of cancer using indocyanine green-labeled monoclonal antibody targeting the epidermal growth factor receptor. Biochem Biophys Res Commun. 2015;464:820-825.

31. Xia J, Kim C, Lovell JF. Opportunities for photoacoustic-guided drug delivery. Curr Drug Targets. 2015;16:571-581.

32. Zhang S, Guo W, Wei J, Li C, Liang XJ, Yin M. Terrylenediimide-based intrinsic theranostic nanomedicines with high photothermal conversion efficiency for photoacoustic imaging-guided cancer therapy. ACS Nano. 2017;11:3797-3805.

33. Muhanna N, Jin CS, Huynh E, et al. Phototheranostic porphyrin nanoparticles enable visualization and targeted treatment of head and neck cancer in clinically relevant models. Theranostics. 2015;5:1428-1443.

34. Yu J, Schuman JS, Lee J-K, Lee SG, Chang JH, Kim K. A light illumination enhancement device for photoacoustic imaging: in vivo animal study. IEEE Trans Ultrason Ferroelectr Freq Control. 2017;64:1205-1211.

35. Ho CJH, Balasundaram G, Driessen W, et al. Multifunctional photosensitizerbased contrast agents for photoacoustic imaging. Sci Rep. 2014;4:5342.

36. Schwarz M, Buehler A, Aguirre J, Ntziachristos V. Three-dimensional multispectral optoacoustic mesoscopy reveals melanin and blood oxygenation in human skin in vivo. J Biophotonics. 2016;9:55-60.

37. Yao Q, Ding Y, Liu G, Zeng L. Low-cost photoacoustic imaging systems based on laser diode and light-emitting diode excitation. J Innov Opt Health Sci. 2017;10:1730003.

38. Daoudi K, van den Berg PJ, Rabot O, et al. Handheld probe integrating laser diode and ultrasound transducer array for ultrasound/photoacoustic dual modality imaging. Opt Express. 2014;22:26365. 